pyelography, and gynaecological investigations and still get neither reassurance nor treatment. Yet it must be remembered that many of these patients look only for reassurance ; the pain is usually not disabling and is likely to resolve spontaneously. Eventually laparotomy may become a diagnostic procedure, and then its advantages and its risks must be assessed on that basis. It is justified when pain persists without diagnosis, and often patients and relatives can be reassured only after the appendix is removed. Nevertheless, when a normal appendix has been removed the general practitioner and the surgeon should not fail to consider other possible causes of the pain.

Chronic appendicitis may or may not exist, but it can be a dangerous diagnosis and deter further thought about the patient. In particular, disordered colonic function can often cause pain in either iliac fossa, and recent work is increasing our knowledge of this phenomenon. ${ }^{3}$ Let us remember above all that the patient would very much like to be relieved of the pain.

\section{Epidemiology of Human Trypanosomiasis in Africa}

African trypanosomiasis (Gambian or Rhodesian sleepingsickness) is an infection by one of the two species of the Trypanosoma brucei subgroup which are infective to mannamely, $T$. gambiense and $T$.rhodesiense. These are usually separated as species though their relationships to $T$. brucei and to each other are debatable. $T$. brucei itself will not infect man, and this characteristic alone distinguishes it from $T$. rhodesiense. T. gambiense, on the other hand, is distinguishable in general by its lesser virulence, causing much more chronic infections and slowly developing disease in man and laboratory animals than $T$. rhodesiense. There is, though, no absolute criterion of distinction, and strains intermediate in both virulence and other characteristics, such as resistance to arsenical drugs, may be encountered. The more chronic, Gambian, form of the disease is the common one in western and western-central Africa, and the more acute, Rhodesian, form occurs on the eastern side of the continent from Bechuanaland and Rhodesia to Uganda and western Kenya.

The disease is transmitted by the bite of an infected tsetse fly (genus Glossina). The fly becomes infective only after trypanosomes ingested in the blood of an infected man or animal have completed their cycle of development and become established in the fly's salivary glands, a cycle which normally takes about three weeks and is completed in only very few flies. Gambian sleeping-sickness is generally associated with riverine tsetse of the $G$. palpalis group and Rhodesian with savannah tsetse of the $G$. morsitans group, though in Kenya there has recently been a striking exception to this rulc in the form of an epidemic of Rhodesian sleepingsickness transmitted by riverine tsetse.

Sleeping-sickness is essentially a disease of rural populations in areas not fully developed, since in country well developed agriculturally or in urban conditions no suitable habitat for tsetse remains and the disease is eradicated with the veztor. Among the most striking features of the epidemiology of the disease are its focal distribution and the fact that its prevalence depends far more on the factors which govern the degree of contact between man and tsetse than on the prevalence of the tsetse itself. Where tsetse are ubiquitous the disease may be rare or non-existent, and yet where tsetse have difficulty in surviving at all, near the drier limits of their geographical distribution, some of the worst epidemics have occurred. The explanation of this apparent anomaly is that in the drier areas the flies become restricted, in the dry season, to isolated pools in the river beds on which the local population are also entirely dependent. Thus a "closed colony" of tsetse are biting and re-biting the same small human population, and ideal conditions are created for the transmission of sleeping-sickness. A single infected fly, in such conditions, having become infective after about three weeks, is potentially a source of another case of sleeping-sickness every time it feeds on human blood, perhaps at three-day intervals, for the rest of its life. Hence, although the proportion of flies infective to man is usually extremely low-far below $1 \%$ - a single infected fly could be the source of ten or even twenty infections. Some such mechanism as this may be responsible for the focal incidence of the disease. A total incidence over a whole population of a small fraction of $1 \%$ may conceal local incidences much higher than this or even single villages where the incidence is $10 \%$ or more, and it is from such foci that a large outbreak can develop rapidly.

The very chronic nature of the disease in typical Gambian sleeping-sickness and the mildness of symptoms in the early stages both contribute to the difficulty of controlling it, particularly in western Africa. Such relatively avirulent strains of trypanosomes are no less transmissible by tsetse, and in a remote African community a period of two years or more may elapse before symptoms, notably of lesions of the central nervous system, become serious enough to attract the attention of first the local people and then the medical authorities. By then the disease may be widespread.

Sleeping-sickness is a disease which requires an effort for its control apparently out of all proportion to its numerical incidence-so long as control measures remain reasonably effective. But if control is relaxed it is capable of explosive outbreak, from a smattering of cases to hundreds of thousands in a few years, and there is a possibility that this process is already gaining momentum in Africa. Since independence many territories have lost experienced workers and sometimes funds have been diverted to other purposes. Avoidable outbreaks have already occurred, but until adequate control measures are restored, particularly over such vast areas as the former Belgian Congo, the risk will remain of epidemics numbered in the tens or hundreds of thousands. The need for suitable training and finance for medical men who are going to work in Africa was recently stressed in these columns. $^{1}$

Another consequence of the chronicity of the disease is of more direct importance in Britain to-day, as may be seen from a letter in the correspondence columns this week (page 167). It is that trypanosomiasis should be borne in mind in the differential diagnosis of symptoms of meninge: 1 or cerebral origin in any African, or European from Africa, particularly if coming from the western side of the continent. The period from first infection to the beginning of severe symptoms may be as much as three years or even longer, especially in a person from places in the most westerly parts of the distribution of the disease such as Guinea, Gambia, or Senegal. The Rhodesian form, from the eastern side of Africa, is usually much more acute, with serious effects on 
the central nervous system beginning within three or four months. Nevertheless, as occasional very chronic cases have been recorded from areas normally associated with Rhodesian sleeping-sickness, the possibility of a diagnosis of trypanosomiasis should never be overlooked.

\section{Cancer Research}

The 42nd Annual Report of the British Empire Cancer Campaign for Research, ${ }^{1}$ published this week, shows that cancer research is active in many directions, and some advances have brought real benefit in recent years. One of the difficulties in cancer research is that cause and effect may be separated for periods of up to 40 or more years. It is not clear why it takes so long for cancer to develop after exposure to a carcinogen, but evidence from biochemical, chromosomal, ${ }^{2}$ and antigenic ${ }^{4}$ studies suggests that a succession of changes occurs during the interval. The immediate biochemical and histological effects of carcinogens on tissues are often quite different from the characteristics which distinguish tumour tissue from normal tissue. Because of this it has, so far, been impossible to point to any one of the immediate effects of a carcinogen as the crucial change which eventually determines the production of cancer.

Recent studies, however, have tended to narrow the gap between cause and effect. Some of the mammalian tumour viruses discovered in recent years-for example, polyoma virus, SV40 virus, adenovirus 12 , or a newly discovered sarcomainducing virus ${ }^{5}$-if injected into suitably susceptible animals, are able to induce neoplasia without delay, visible tumours appearing within days of injection of the virus. Susceptible normal cells maintained in tissue culture may be transformed instantaneously to "cancer-like" cells by the introduction of an oncogenic virus into the culture medium. ${ }^{6} 7$

Unfortunately the detailed chemical structure of the nucleic acids of viruses is unknown, so that we are still unable to pinpoint the chemical mechanism involved. If, however, claims to have transformed cells by exposing them in vitro to chemical carcinogens ${ }^{8}$ can be substantiated, then there is hope of real progress. In this connexion the greatly improved methods for preparing and analysing nucleic acids and proteins ${ }^{9}$ will play a dominant role.

Knowledge of the causes of cancer and of methods of preventing it is accumulating all the time. For instance, evidence that asbestos is a serious hazard is mounting. ${ }^{10}$ The fact that exposure to it gives rise specifically to pleural or peritoneal mesothelioma is remarkable. The possibility that it is more than a minor cause of lung cancer is a matter for considerable concern. But epidemiological and experimental studies by cancer research workers can do no more than point to hazards. Co-operation by the public, by industry, by the weight of medical opinion, and by the Government is essential if progress is to be made in the prevention of the cancers.

Further research has continued into methods for detecting precancerous lesions, or diagnosing invasive cancer at a stage when eradication is feasible. From the report it seems that experience in Britain ${ }^{11}$ with mammography in diagnosis of breast cancer has been less encouraging than in the United States. Satisfactory yet quicker and cheaper substitutes for cytological examination in cases of cancer and pre-cancer of the human cervix have not yet been found. ${ }^{12-1.5} \mathrm{~J}$. W. D.
Bull and J. Marryat ${ }^{16}$ at the National Hospital, Queen Square, London, have investigated the locating of brain lesions by means of radioactive isotopes. They conclude that because the method is free from risk of trauma and can be carried out in the out-patients department it is preferable to angiography and pneumoencephalography as the first major "special" investigation. A drawback of the method is the occurrence of false-negative results, though false-positive results have not been encountered. Meningiomata tend to have a distinctive appearance under the scanner, and the existence of a multiplicity of secondary deposits has been revealed by this method of investigation after having been missed by other methods.

If the aim of cancer treatment is to prolong life indefinitely, and if it is to be regarded as successful only when neoplastic tissue is completely eradicated, then relatively little advance has been made during recent years. But this misrepresents the situation. The development of painless diagnostic procedures, advances in surgery and anaesthesia, and improvements in radiotherapy, particularly through the use of computers in the planning of treatment and of better ways of delivering treatment from high-energy sources of radiation, have led to spectacular improvements in treatment. Suffering from cancer, though not always death from it, has been dramatically reduced. Useful and enjoyable life, but not always the chronological length of life, has been greatly prolonged.

M. E. Whisson and T. A. Connors ${ }^{17}$ found that some chemotherapeutic agents were far more effective than others in inhibiting the growth of a transplantable plasma-cell tumour in mice. Aniline mustard (CB.1074) was found to be exceptionally effective, and cases of human multiple myeloma are at present being treated with this substance in a controlled clinical trial. Striking success in the treatment of trophoblastic tumours by combinations of methotrexate and 6-mercaptopurine are reported from Charing Cross Hospital, ${ }^{18} 39$ out of 45 patients being in complete remission and six of these having had successful normal pregnancies after chemotherapy. Developmental abnormalities in the offspring were not observed.

A great debt of gratitude is due to all those who contribute to the achievements of the British Empire Cancer Campaign and to the other charitable organizations which support cancer research. At the same time it is becoming increasingly apparent that unless substantial financial aid is supplied by the Government this country is going to play no more than a minor part in new developments, which require computer time and special facilities for the study of, for example, infective viruses and the effects of potentially carcinogenic agents on non-human primates.

\footnotetext{
British Empire Cancer Campaign for Research, Forty-second Annual Report covering the year 1964. 1965.

Koller, P. C., Wallis, V., and Talukdar, C., ibid., 63.

Koller, P. C., W nat. Cancer Inst., 1963, 31,791.

Molomut, N., Neoplasma (Bratisl.), 1963, 10, 155.

Harvey, J. J., Rep. Brit. Emp. Cancer Campgn, 1964, 42, 185. Halberstaedter, L., Dolfanski, L., and Tenenbaum, E., Brit. f. exp. Vogt, M., and Dulbecco, R., Proc. nat. Acad. Sci. (Wash.), 1960, 46, 365.

Berwald, Y., and Sachs, L., Nature (Lond.), 1963, 200, 1182.

Hastings, J.' R. B., and Kirby, K. S., Rep. Brit. Emp. Cancer Campgn, $1964,42,3$.

10 Brit. med. F., 1964, 2, 202. . I. R., Rep. Brit. Emp. Cancer Campgn, 1964, 42, 309.

12 Spector, W. G., ibid., 252.

13 MacGillivray, I., ibid., 295

14 Cameron, C. B., and Weg, M. W., ibid., 119.

is Nixon, W. C. W., Labrum, A. H., and Gibbs, D. E., ibid, 304.

Bull, J. W. D., and Marryat, J., ibid, 610.

Whisson, M. E., and Connors, T. A., ibid., 71.

Bagshawe, K. D., Wilde, C. E., Brewis, R. A. L., and de Wardener, H. E., ibid., 679.
} 